\title{
THE RELIABILITY AND VALIDITY OF THE TURKISH VERSION OF THE EUROPEAN HEALTH INTERVIEW SURVEY - PHYSICAL ACTIVITY QUESTIONNAIRE (EHIS-PAQ)
}

\author{
Mehmet Ozkeskin ${ }^{1}$, Fatih Ozden²
}

${ }^{1}$ Ege University, Faculty of Health Sciences, Physical Therapy and Rehabilitation Department, İzmir, Turkey
2 Muğla Sıtkı Koçman University, Köyceğiz Vocational School of Health Services, Elderly Care Department, Muğla,
Turkey

Address for Correspondence: Fatih Özden PT. MSc. E-mail: fatihozden@mu.edu.tr Received: 02.02.2021; Accepted: 20.05.2021; Available Online Date: 27.05.2021

(C) Copyright 2021 by Dokuz Eylül University, Institute of Health Sciences - Available online at https://dergipark.org.tr/en/pub/jbachs

Cite this article as: Ozkeskin M,Ozden F. The reliability and validity of the Turkish version of the European Health Interview Survey - Physical Activity Questionnaire (EHIS-PAQ) J Basic Clin Health Sci 2021; 2: 124-133.

\begin{abstract}
Purpose: To translate and adapt the Turkish version of the EHIS-PAQ and to evaluate its reliability and validity in young adults.

Methods: A total of 431 young adults were assessed with the Turkish version of the EHIS-PAQ and International Physical Activity Questionnaire-Short Form (IPAQ-SF). One week later, 117 participants refilled the Turkish EHIS-PAQ for the test-retest reliability. Reproducibility and construct validity was analyzed with intraclass correlation coefficient (ICC) and Pearson correlation coefficient, respectively. Besides, standard error of measurement (SEM95) and minimal detectable change (MDC 95 ) were calculated for the Turkish EHIS-PAQ.

Results: The mean age of the participants were $21.2 \pm 9.8$ years. The ICC score for the total Physical Activity (PA) of Turkish EHIS-PAQ was 0.873 (Cl:0.81-0.91). Test-retest reliability for the total score was excellent $(I C C>0.80)$. The highest correlation was observed between the walking minute value of Turkish EHIS-PAQ and the walking PA sub-score of IPAQ-SF, as expected $(r=0.625, p<0.001)$. There was also a strong correlation between the Transportation-related PA subscore of the Turkish EHIS-PAQ and IPAQSF's Walking PA score $(r=0.592, p<0.001)$. SEM95 and $\mathrm{MDC}_{95}$ for the Transportation-related PA were 172.5 and 476.8, respectively.

Conclusion: The Turkish version of the EHIS-PAQ is a valid and reliable questionnaire in young adults.
\end{abstract}

Keywords: EHIS-PAQ; reliability; Turkish version; validity

\section{INTRODUCTION}

Deaths due to physical inactivity ranks fourth among risk factors worldwide (1). Although the causes of these deaths due to physical inactivity are diseases such as arm cancer, ischemic stroke, and breast cancer, physical inactivity is a variable risk factor. Inadequate physical activity is the 4 th foremost risk determinant for early mortality in Europe and ranked among the world's highest ten causes $(2,3)$.

Physical activity levels vary according to each community. Age, gender, education level, and socioeconomic levels of countries affect the level of physical activity. The use of physical activity assessment questionnaires diverges according to the 
characteristics of the target population (4). Scales developed for children, young people, and old people are frequently used in the field. According to the results of these scales, it is aimed to provide changes in body structure, activity, and participation level within the framework of International Function Classification by directing individuals to appropriate and sufficient physical activity. It is important that the physical activity scales to be chosen to assess the capacity of individuals are appropriate to the characteristics of the society, environment, habits, and lifestyle $(5,6)$.

Different methods, both subjective and objective, are used to measure the level of physical activity (7). Surveys differ according to how physical activity parameters are obtained (8). In the questionnaires to collect for the level of physical activity, it is difficult to compare different populations and there is no methodological quality (9). There are surveys in the literature that measure the physical activity levels of people in different societies. Among these, the International Physical Activity Questionnaire (IPAQ) was designed as a short and long-form to identify physical activity and sedentary lifestyles of adults (10). The Global Physical Activity Questionnaire (GPAQ) questionnaire was developed by the World Health Organization in order to evaluate the physical activity status in different countries $(1,11)$. This survey is an International Physical Based on the Activity Scale, it has been brought to the field and has been shown to have reliability. This questionnaire has also been validated and is used very often $(1,10,11)$.

Based on data collected for the first cycle population of the European Health Interview Survey (EHIS) in
European Union member states between the ages of 2006 and 2010, the first wave of EHIS posed major difficulties during data collection with the short version IPAQ $(12,13)$. Expert organizations and cognitive measurement researchers have proven significant difficulties in learning various physical activity intensity degrees, stating the duration of regular movements (e.g., walking or sitting and combining multiple activities. As a result, the EHIS Core Group has developed the "EHIS-Physical Activity Questionnaire (PAQ)", a field-specific physical activity questionnaire (13). So far, the Turkish version of this questionnaire has not been culturally adapted and validated. The aim of this study is to demonstrate the psychometric properties of the Turkish version of the EHIS-PAQ questionnaire among young adults.

\section{METHODS}

\section{Translation and Adaptation Process}

Firstly, permission was received from Finger JD, who developed EHIS-PAQ, to create the Turkish version of the scale and to carry out the validity and reliability study (13). In the process of creating the Turkish version of EHIS-PAQ and investigating its validity and reliability, Guillemin et al. and Beaton et al.'s intercultural adaptation methods and recommendations were taken into account $(14,15)$. The questionnaire was first translated from English to Turkish independently from each other by two translators whose native language is Turkish and has a good English command. Then, the translation committee synthesized these translations according to linguistic and cultural characteristics. Turkish translations have been translated into English by two

Table 1. The characteristics of the participants

\begin{tabular}{ll}
\hline $\mathrm{n}: 431$ & Total \\
\hline Age (years, mean \pm SD) & $21.2 \pm 9.8$ \\
Gender $(\mathrm{n}, \%)$ & $374(86.8)$ \\
Women & $57(13.2)$ \\
Men & $21.4 \pm 3.4$ \\
Body Mass Index $\left(\mathrm{kg} / \mathrm{m}^{2}\right.$, mean $\left.\pm \mathrm{SD}\right)$ & \\
Living condition $(\mathrm{n}, \%)$ & $379(87.9)$ \\
Family & $4(0.9)$ \\
Relatives & $22(5.1)$ \\
Alone & $26(6.0)$ \\
Friends & \\
Residence $(\mathrm{n}, \%)$ & $376(87.2)$ \\
Home & $55(12.8)$ \\
Dormitory & \\
\hline
\end{tabular}

SD: standard deviation, n: number of patients 
independent translators without any information about the questionnaire. Both translations were compared with the original version of EHIS-PAQ. Afterward, a single Turkish version was created and examined in terms of conceptual inaccuracies and inconsistencies, considering Turkish society's sociocultural structure. The final Turkish version of the scale was created. In order to evaluate the understandability of the Turkish EHIS-PAQ, it was pretested in a pilot study on randomly selected 30 young adults. The comprehensibility of each item of the Turkish EHIS-PAQ was tested with the Likert scale (consisting of five answers) whether there were any incomprehensible parts. After the Turkish version of EHIS-PAQ was found to be understandable, the application phase of the study was initiated.

\section{Study Design and Participants}

This study was carried out on 431 young adults. The inclusion criteria of the study were Turkish speaking and individuals aged over 18 years. Individuals with chronic diseases and who did not want to participate voluntarily were excluded from the study. The individuals' sociodemographic status (e.g., age, gender, body mass index, residence) were recorded. Participants were evaluated with the Turkish version of the EHIS-PAQ, and IPAQ-SF in the first assessment. A retest evaluation was performed one week later by applying the Turkish version of EHIS$P A Q$ to 117 individuals. The minimum sample size required for statistical analysis was calculated using the $\mathrm{G}^{*}$ Power 3 (Universitat Dusseldorf, Dusseldorf, Germany) computer software $(16,17)$. Considering the correlation coefficients in the German validation study of EHIS-PAQ, the coefficient of determination was set as 0.02 (12). A total of 430 cases were calculated to be required in the study with $90 \%$ power and 0.05 significance level. As a result, we included 431 young individuals in the study. Also, in the calculation for test-retest reliability (Effect size $=0.3$, a significance level $=0.05$ and the power of 0.95 ), at least 111 individuals were required to be adequate. Accordingly, 117 persons were re-evaluated one week later.

\section{European Health Interview Survey - Physical Activity Questionnaire (EHIS-PAQ): EHIS-PAQ is an 8-item questionnaire developed by the European Union's EHIS core group as an alternative to other physical activity assessment tools. The scoring algorithm of the questionnaire was presented in the development study by Finger et al (13). Including Likert-type answers especially for some items increases the answering of the questionnaire. EHIS- $\mathrm{PAQ}$ questions the weekly physical activity level during work, transportation, and leisure activities and}

Table 2. Absolute values (mean, standard deviation, percentile, min-max) of the participants

\begin{tabular}{llll}
\hline $\mathrm{n}: 431$ & & Mean \pm SD & Range \\
\hline EHIS-PAQ & & \\
& Work-related PA & $1.64 \pm 0.53$ & $(1-3)$ \\
& Walking minute & $164.95 \pm 195.16$ & $(0-1260)$ \\
& Cycling time & $6.79 \pm 27.61$ & $(0-315)$ \\
& Transportation-related PA & $585.13 \pm 674.10$ & $(0-4158)$ \\
& Aerobic PA & $75.52 \pm 134.27$ & $(0-1500)$ \\
& & & \\
& Health-enhancing PA & $1.85 \pm 0.35$ & $(1-2)$ \\
& Muscle-strengthening PA & $1.65 \pm 0.47$ & $(1-2)$ \\
& Total PA & $1.53 \pm 0.49$ & $(1-2)$ \\
& & & $(0-4851)$ \\
& Walking PA & $614.35 \pm 722.38$ & $(0-11760)$ \\
& Moderate PA & $279.07 \pm 845.39$ & $(0-7680)$ \\
& Vigorous PA & $284.64 \pm 771.58$ & $(30-2100)$ \\
& Sitting time & $398.43 \pm 237.42$ & $(120-13506)$ \\
\hline
\end{tabular}

SD: standard deviation, $n$ : number of patients, EHIS-PAQ: Turkish version of the European Health Interview Survey Interview-Physical Activity Questionnaire, PA: Physical Activity, IPAQ-SF: International physical Activity Questionnaire-Short Form 
also include Likert-type answers for some items, which increases the answering of the questionnaire $(12,13)$. A high score of "Walking minute, Cycling time, Transportation-related PA, Aerobic PA" indicate a high physical activity level of the individuals. On the other hand, a high score of "Work-related PA, Healthenhancing PA, Muscle-strengthening PA, Total PA" indicate a low physical activity level.

International Physical Activity QuestionnaireShort Form (IPAQ-SF): IPAQ-SF was developed with the support of the World Health Organization (WHO) and the United States Centers for Disease Control and Prevention (CDC) $(18,19)$. Turkish validity and reliability study made by Sağlam et al. in Turkey (20). It provides information about the time and sitting times, people spend in light, medium, and severe activities. When evaluating the activities, the criterion is to conduct each activity for at least ten minutes. The "MET-min / week" score is obtained by multiplying the MET value (metabolic equivalent) days and minutes for each activity level. As determining how much energy is used for physical activity, each activity's hebdomadal span in minutes is multiplied by the BAT values determined for IPAQ-
SF (21). As a result, the energy consumed by the individuals at the light, moderate, and severe activity level and the total activity level will be calculated (22).

\section{Statistical Analysis}

SPSS for Windows v25.0 (SPSS Inc, Chicago, IL, USA) software was used for the analysis. The mean and standard deviation (SD) were given for the quantitative parameters. Percentage distribution is presented for qualitative data. The homogeneity of the participants was calculated with the Shapiro-Wilk test. The confidence interval was accepted as 0.95 .

\section{Reliability}

In the reproducibility analysis of the Turkish EHIS$P A Q, I C C$ coefficient and $\mathrm{Cl}$ values were calculated for subscores (Work-related PA, Transportationrelated PA, Health-enhancing PA, Musclestrengthening $P A)$, total score, walking minute, cycling time and Aerobic PA. In addition, Standard error of measurement (SEM) and Minimal detectable change (MDC) values were calculated for all these standard values. The MDC value was determined to provide the minimally detectable actual change score of the Turkish EHIS-PAQ rather than the

Table 3. Test-retest reliability of the Turkish version of the European Health Interview Survey Interview Physical Activity Questionnaire (EHIS-PAQ)

\begin{tabular}{|c|c|c|c|c|c|}
\hline $\mathrm{n}: 117$ & Test (Mean \pm SD) & $\begin{array}{l}\text { Retest } \\
(\text { Mean } \pm \text { SD) }\end{array}$ & $\mathrm{ICC}(95 \% \mathrm{Cl})$ & $\mathrm{SEM}_{95}$ & $\mathrm{MDC}_{95}$ \\
\hline \multicolumn{6}{|l|}{ EHIS-PAQ } \\
\hline Work-related PA & $1.65 \pm 0.49$ & $1.65 \pm 0.47$ & $\begin{array}{l}0.921(0.88- \\
0.94)\end{array}$ & 0.13 & 0.38 \\
\hline Walking minute & $167.60 \pm 152.67$ & $163.24 \pm 162.19$ & $\begin{array}{l}0.904(0.86- \\
0.93)\end{array}$ & 47.0 & 130.1 \\
\hline Cycling time & $4.95 \pm 16.10$ & $4.78 \pm 13.47$ & $\begin{array}{l}0.648(0.49- \\
0.75)\end{array}$ & 9.4 & 26.2 \\
\hline Transportation-related PA & $582.84 \pm 525.95$ & $567.43 \pm 547.25$ & $\begin{array}{l}0.892(0.84- \\
0.92)\end{array}$ & 172.5 & 476.8 \\
\hline Aerobic PA & $102.09 \pm 179.39$ & $80.38 \pm 84.96$ & $\begin{array}{l}0.442(0.19- \\
0.61)\end{array}$ & 133.7 & 369.4 \\
\hline Health-enhancing PA & $1.82 \pm 0.38$ & $1.82 \pm 0.38$ & $\begin{array}{l}0.745(0.63- \\
0.82)\end{array}$ & 0.19 & 0.53 \\
\hline Muscle-strengthening PA & $1.59 \pm 0.49$ & $1.64 \pm 0.48$ & $\begin{array}{l}0.820(0.74- \\
0.87)\end{array}$ & 0.20 & 0.57 \\
\hline Total PA & $1.55 \pm 0.49$ & $1.58 \pm 0.49$ & $\begin{array}{l}0.873(0.81- \\
0.91)\end{array}$ & 0.17 & 0.48 \\
\hline
\end{tabular}

n: number of patients, ICC: Intra-class correlation coefficient, Cl: Confidence interval, EHIS-PAQ: Turkish version of European Health Interview Survey Interview Physical Activity Questionnaire, PA: Physical Activity SEM: Standard error of measurement; MDC: Minimal detectable change. 
measurement bias or error. The formula $\mathrm{MDC}_{95}=$ $1.96 * S E M * \sqrt{ } 2$ and $S E M 95=S D * \sqrt{ }(1-I C C)$ are used for SEM and MDC calculations, respectively (23).

\section{Validity}

The Pearson correlation coefficient $(r)$ between the Turkish EHIS-PAQ and International Physical Activity Questionnaire-Short Form (IPAQ-SF) was calculated in the construct validity. The correlation was analyzed for both subscores and total scores. A high correlation was expected within the scope of convergent validity among similar subscores and low correlation was expected in terms of discriminant validity between structurally different subscales. Correlational coefficients were interpreted as excellent if $>0.5$; as moderate if between 0.5 and 0.35 ; and low if $<0.35$ (24). We also performed the Bland-Altman plot to demonstrate the agreement between the transportation-related PA subscore of the Turkish EHIS-PAQ and the walking PA of the IPAQ-SF (25). Since both subscores represent the transportation amount of individuals as "MET/min-per week" both were used as a plot analysis model $(13,20)$. The mean bias and the $95 \%$ limits of agreement are plotted to provide the difference between the calculations.

\section{RESULTS}

A total of 431 participants ( 374 female, 57 male) with a mean age of $21.2 \pm 9.8$ were enrolled in the study. The mean body mass index was $21.4 \pm 3.4$. The majority of the participants $(87.9 \%)$ live with their families. Also, most of them $(87.2 \%)$ were home residents. The physical and demographic characteristics of the participants are presented in Table 1. The average scores of the Turkish EHISPAQ and IPAQ-SF are presented in Table 2. No changes were required in the Turkish EHIS-PAQ after pretest for cultural adaptation and during translation procedures. All items of the questionnaire were suitable in terms of comprehensibility.

\section{Reliability}

In Table 3, test and retest means, ICC scores, $\mathrm{Cl}$ values, SEM and MDC of Turkish EHIS-PAQ's subscores, total score and other standardized reference scores are presented. The ICC score for the total PA of Turkish EHIS-PAQ was 0.873 (Cl:0.81$0.91)$. Test-retest reliability for the total score was excellent (ICC $>0.80)$ (Table 3$)$. All scores are found to be reliable (ICC>0.60, ranged between 0.648 to 0.921 ), except aerobic PA (ICC $=0.442$, Cl:0.16-

Table 4. Construct validity of the Turkish version of the European Health Interview Survey Interview Physical Activity Questionnaire (EHIS-PAQ)

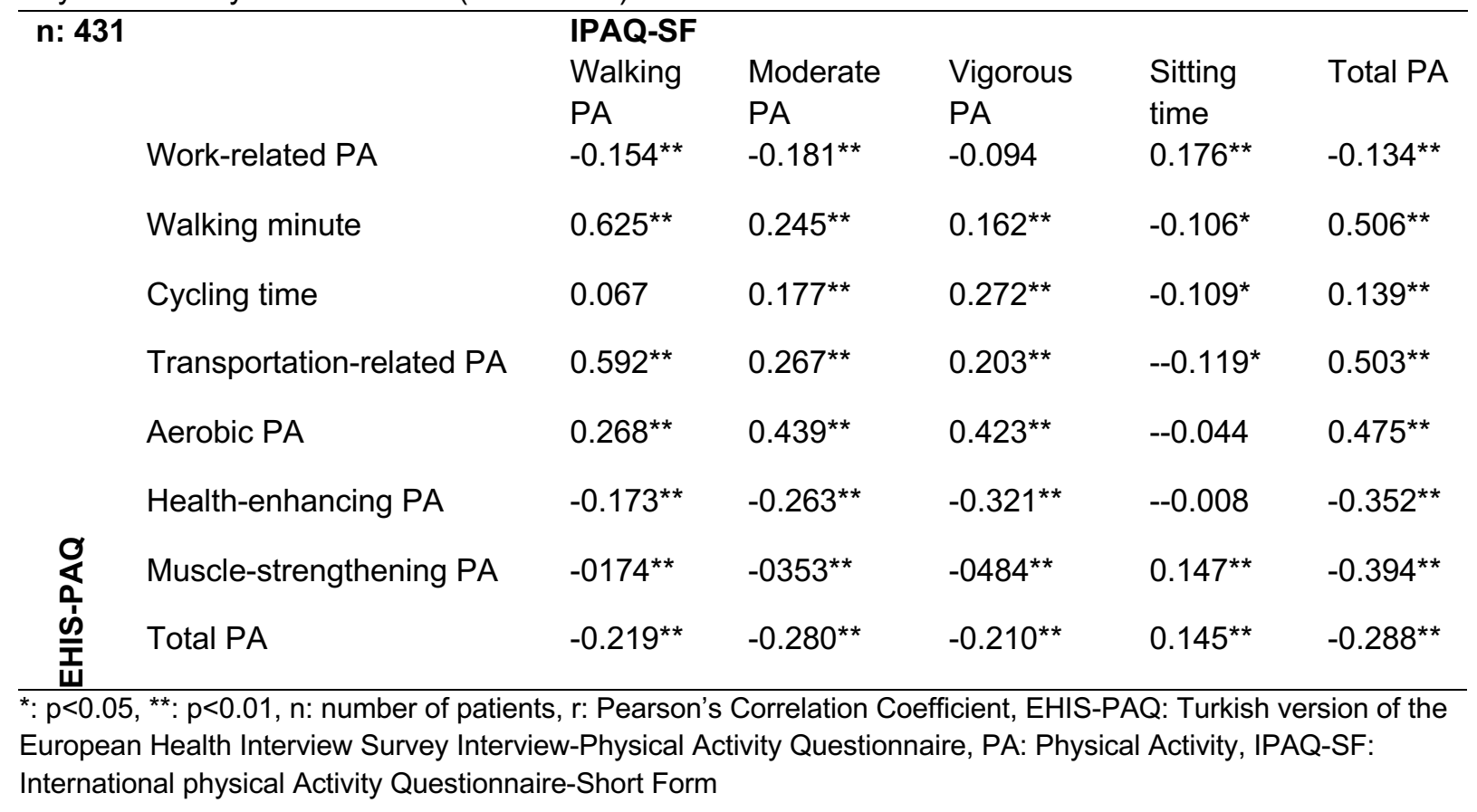


0.61)). SEM 95 and $\mathrm{MDC}_{95}$ for the total Transportationrelated PA score were 172.5 and 476.8 , respectively.

\section{Validity}

The Pearson correlation coefficient calculated between the scores of Turkish EHIS-PAQ and IPAQ$\mathrm{SF}$ is presented in Table 4. The highest correlation was observed between the walking minute value of Turkish EHIS-PAQ and the walking PA sub-score of IPAQ-SF, as expected $(r=0.625, p<0.001)$. There was also a strong correlation between the Transportationrelated PA subscore of the Turkish EHIS-PAQ and IPAQ-SF's Walking PA score $(r=0.592, p<0.001)$. In addition, a strong correlation was found between the walking minute value of Turkish EHIS-PAQ and the total PA score of IPAQ-SF $(r=0.506, p<0.001)$. Besides, a strong correlation was observed between Turkish EHIS-PAQ's Transportation-related PA subscore and the total PA value of IPAQ-SF $(r=0.503$, $\mathrm{p}<0.001)$. Another moderate-strong relation was proven between the Aerobic PA value of Turkish EHIS-PAQ and the total PA score of IPAQ-SF, as expected $(r=0.475, p<0.001)$. Also, as expected, a moderate-strong correlation was found between the Muscle-strengthening PA sub-score of Turkish EHISPAQ and the Vigorous PA sub-score of IPAQ-SF ( $r=$ $0.484, \quad p<0.001)$. Relationships between other coefficients were low and moderate correlated $(0.08$ to 0.439), conforming to the convergent and discriminant validity. The Bland-Altman plot of the agreement for the Turkish version of the EHIS-PAQ's Transportation-related PA subscore and the IPAQSF's Walking PA was presented in Figure-1. The mean difference was small as expected (-29.21 MET / min-per week, $p>0.05$ ). $95 \%$ limits of agreement were -1281.5 to 1223.0 , wide ranging (Figure-1).

\section{DISCUSSION}

The results of the study were showed that the Turkish version of the EHIS-PAQ is a reliable and valid questionnaire for the evaluation of physical activity in Turkish-speaking young adults. EHIS-PAQ is a short, practical, short time-filled and pragmatic physical activity assessment tool due to its domain-specific nature. In the evaluation of specific groups in public health assessments, it can provide a more comprehensive assessment for its domains of different aspects (e.g., work, translation, aerobic, muscle-strengthening) $(12,13)$. On the other hand, these sub-scores are not scored in IPAQ. The physical activity level is only scored in IPAQ for low, moderate and vigorous activities (20). Althougt as the commonly preferred questionnaires, IPAQ and Global Physical Activity Questionnaire (GPAQ) is developed specifically for use in low and middleincome countries $(1,11,13)$. On the contrary, EHIS$\mathrm{PAQ}$ is developed for the assessment of participants mostly living in high-income countries in order to evaluate the level of physical activity more clearly (13). Also, EHIS-PAQ includes some Likert-typed answers, which can provide a quicker and more practical scoring. Besides, the scores to be obtained can provide a more homogeneous and standardized data. Calculation of EHIS-PAQ can be done practically by setting up a few simple algorithms. It can be calculated quickly due to its 8-item structure and can provide quick monitoring results of individuals.

The original questionnaire developed by the EHIS core group in English (13). The development of the questionnaire was inspired by IPAQ, GPAQ, US national health interview survey, and US behavioral risk factor surveillance survey $(12,13)$. EHIS-PAQ has been developed due to the insufficient competence of IPAQ during the focus group interviews and cognitive tests of EHIS waves. The psychometric properties of the EHIS-PAQ were investigated only in German (12). In our study, EHIS-PAQ was translated into Turkish and adapted, also its psychometric properties were analyzed. One of the unique aspect of the present research is that the SEM and MDC values were presented for the first time for EHIS-PAQ. MDC value is essential in monitoring the lowest significant change in the physical activity levels of individuals (26). In addition to epidemiological studies involving public health, case-controlled, and randomizedcontrolled studies can also benefit from the MDC value of our study.

In our study, the international translation procedures recommended were used to translate EHIS-PAQ from English to Turkish $(14,15)$. In the English development study of EHIS-PAQ, it was stated that the questionnaire was translated into French, Estonian, German with generally accepted translation procedures, and adaptation was carried out by applying a pilot test. The final versions of the questionnaires were obtained by taking into account the linguistic and cultural characteristics of the relevant language by pretesting between 35-50 individuals for translation into each language (13). In our study, a pilot test was conducted with 30 participants for pretest after translation procedures. In 
Figure 1. Bland-Altman plot for the agreement of transportation-related PA from the Turkish version of the European Health Interview Survey Interview Physical Activity Questionnaire (EHIS-PAQ) and the Walking PA from the IPAQ-SF (MET/min-per week, $\mathrm{n}=431$ )

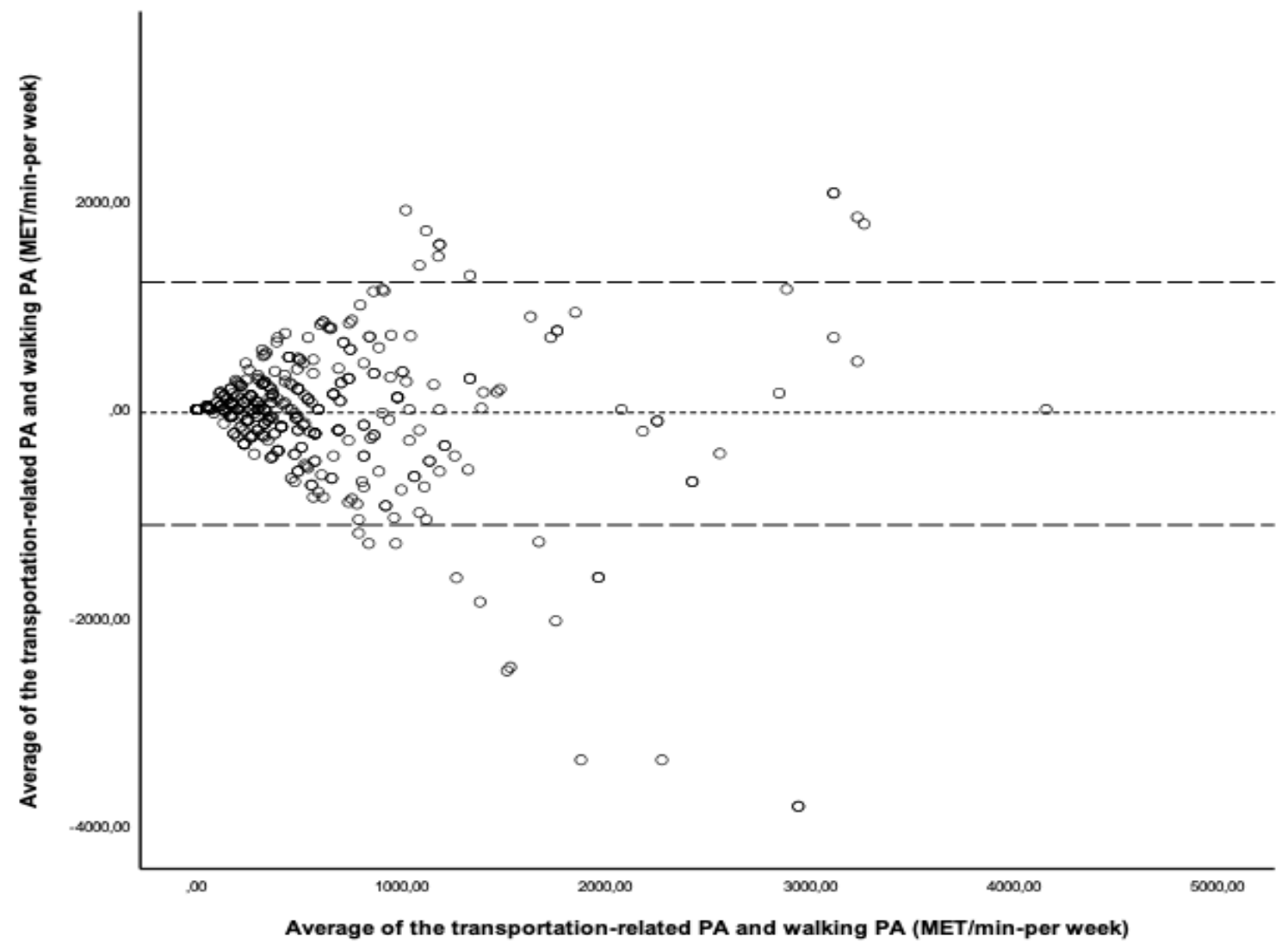

this test, the understandability of the questionnaire was measured. Since there was no difficulty in terms of comprehensibility, no grammatical change was required.

The German validation study was carried out with 140 people between the ages of $15-79$ (12). Our study was conducted with 431 young adult individuals (21.2 \pm 9.8$)$. The reason for evaluating young adults in our sample was to obtain a more homogeneous sample. Because, since EHIS-PAQ contains specific domains, it may be possible to observe in which of these domains the physical activity orientation of the youth is concentrated. In addition, different cut-off values were used for 15-17 years of age in the scoring of EHIS-PAQ (12,13). Therefore, we evaluated individuals $>18$ years. In our study, test-retest reliability evaluated with one-week intervals for the reproducibility of the questionnaire. ICC was calculated for Turkish EHIS-PAQ's subscores, total score, and other reference values. All subscales and total score of the questionnaire were reliable $(0.745-$ 0.921).

However, Aerobic PA, one of the reference values calculated, was found to be low-level reliable (0.442). This score is calculated by adding cycling time in addition to the time spent on sports, fitness, and recreational activities questioned in the 7 th question. The lack of regular cycling habits in Turkey might have provided unclear answers between the two assessments. Minute values of regular activity may be expressed more clearly, but minute expressions related to habits that have not yet been acquired may not be clearly expressed. The ICC score of 0.648 calculated for cycling time also supports our related views. In the German version study, the questionnaire was applied twice with an interval of 30 days (12). In the generally accepted recommendations, it was stated that the questionnaires should be repeated with a minimum of 2 days and a maximum of 2 weeks for retesting. The shorter period could bring about 
cognitive problems or longer periods may cause actual variational problems (27). Accordingly, we preferred a one-week interval in our study. In the German EHIS-PAQ, retest performed with 123 people, ICC values were calculated for similar scores to our study and it was stated that the value ranged between 0.43 and $0.73(12)$. It is seen that the ICC calculated for cycling time is 0.53 and a value close to our study was obtained. Common statistical guidelines predicted that ICC values above 0.5 are acceptable, (28) then can state that the Turkish version of EHIS-PAQ is reliable.

In our study, the construct validity was checked in terms of both convergent and discriminant validity. We compared the Turkish EHIS-PAQ with the most widely used physical activity PROMs, IPAQ-SF $(12,20)$. We did not use an accelerometer in validation. The fact that pedometers did not give reliable results was the first reason for this decision $(29,30)$. Secondly, we thought that PROMs should be compared with each other as a subjective evaluation tool (27). In the German validation study, the long form of IPAQ was preferred (12). Since IPAQ-SF is a 7-item tool, we thought that it would be more appropriate to compare with EHIS-PAQ in our study (20). It was also obvious that too many survey questions would be a burden for the patient (31). According to our results, Turkish EHIS-PAQ and IPAQ-SF walking indicators were compatible $(r=$ $0.625, p$ <0.001). Similarly, Turkish EHI-PAQ's Transportation-related PA sub-score was strongly correlated with the walking indicator of IPAQ-SF $(r=$ 0.592, $p<0.001$ ). In addition, the walking minute indicator of Turkish EHIS-PAQ was highly correlated with the IPAQ-SF total PA score $(r=0.506, p<0.001)$. The Transportation-related PA subscore of Turkish EHI-PAQ and the total PA value of IPAQ-SF were also strongly correlated $(r=0.503, p<0.001)$. These values, which were expected to be highly correlated in terms of convergent validity, were associated with each other at an acceptable level for validity. Relationships between other score's coefficients were low and moderate correlated (0.08 to 0.439 ), conforming to both convergent and discriminant validity. The highest correlation coefficient for convergent validity was 0.625 . The absence of higher correlations may have been due to calculation differences between the two surveys. With its domain-specific structure, categorical subscores were obtained in EHIS-PAQ $(12,13)$. Some of EHIS$P A Q$ items are scored between 1-3 and some others with 1-2. In the IPAQ-SF, the correlation coefficient may not be as high as expected because calculations are scored in MET/minutes per week. In the German validation study, the correlation coefficients between the EHIS-PAQ and the IPAQ long form were between 0.01 and 0.64 , and both are highly similar to our study (12). According to these results, we can state that the construct validity of the Turkish EHIS-PAQ in our study is appropriate.

On the other hand, we developed a model for validity by the Bland-Altman plot of the agreement technique with Turkish EHIS-PAQ's transportation-related PA subscore and the IPAQ-SF's Walking PA (25). The mean difference was small as expected (-29.21 MET / min-per week, $p>0.05$ ). $95 \%$ limits of agreement were -1281.5 to 1223.0 , which has been considered as wide. As can be seen in the Bland-Altman graph (Figure-1), it is seen that the distribution of differences is wider as the average scores in the measurements increase. This situation showed that the measurement variation of the questionnaire depends on the size of the results.

In our study, SEM and MDC value for EHIS-PAQ has been demonstrated for the first time. We calculated the SEM and MDC values with generally accepted statistical formulas. We used the ICC and standard deviation values in this calculation. According to our results, SEM and MDC values were 172.5 and 476.8, respectively. SEM demonstrates the smallest change that specifies an actual difference for the whole sample. In addition, MDC represents the minimal change that shows a real progression for a singular case $(26,32)$. In particular, SEM value is essential for epidemiological and cohort studies, and MDC for physical activity monitoring in case-control and randomized controlled studies.

The limitations should also be acknowledged. Firstly, the sample in our study consisted of young adult individuals. Studying on a homogenous group in validity and reliability studies would provide the statistical analysis results stronger. However, this situation restricts the generalizability of our results to the elderly Turkish population. Secondly, we did not perform concurrent validity analysis due to the low reliability of the accelerometer and pedometer devices $(29,30)$. However, correlation with objective physical activity data could also make our results more meaningful in another respect. Considering that EHIS-PAQ was specially developed for high-income countries, selecting the study sample from the different income groups of individuals from different 
cultural segments would be more efficient in terms of cross-cultural adaptation $(12,13)$. Finally, using GPAQ in addition to IPAQ-SF could further diversify our construct validity results $(1,11)$. However, applying three separate questionnaires containing similar questions to patients at the same time could raise concerns on data collection. Also, directing a large number of questions could burden the participants (31).

\section{CONCLUSION}

The Turkish version of the EHIS-PAQ is a valid and reliable questionnaire in young adults. SEM and MDC provide an essential reference value for monitoring the physical activity. Further research should focus on the responsiveness of Turkish EHIS-PAQ with sensitive accelerometer-based evaluation.

Ethics Committee Approval: The permission for the translation for the Turkish version of EHIS-PAQ was acquired from the developer of the original questionnaire. The study was carried out in accordance with the ethical principles and the Helsinki Declaration. The study protocol was approved by the ethics committee of Ege University (No: 20-11.1T/37)

Informed Consent: Informed consents of the patients were obtained.

Conflict of Interest: None

Peer-review: Externally peer-reviewed.

\section{REFERENCES}

1. Armstrong T, Bull F. Development of the world health organization global physical activity questionnaire (GPAQ). J Public Health. 2006;14(2):66-70.

2. Ezzati M, Riboli E. Behavioral and dietary risk factors for noncommunicable diseases. $\mathrm{N}$ Engl $\mathrm{J}$ Med. 2013;369(10):954-64.

3. Hallal PC, Martins RC, Ramírez A. The Lancet physical activity Observatory: promoting physical activity worldwide. Lancet. 2014;9942(384):4712.

4. Can S. Physical Activity Measurement: Objective and Subjective Methods. Turk. J. Sports Med. 2019;54(4).

5. Helmerhorst HHJF, Brage S, Warren J, Besson $\mathrm{H}$, Ekelund $\mathrm{U}$. A systematic review of reliability and objective criterion-related validity of physical activity questionnaires. Int J Behav Nutr Phys Act. 2012;9(1):103.

6. Albanes D, Conway JM, Taylor PR, Moe PW, Judd J. Validation and comparison of eight physical activity questionnaires. Epidemiology. 1990:65-71.
7. Strath SJ, Kaminsky LA, Ainsworth BE, Ekelund U, Freedson PS, Gary RA, et al. Guide to the assessment of physical activity: clinical and research applications: a scientific statement from the American Heart Association. Circulation. 2013;128(20):2259-79.

8. Prince SA, Adamo KB, Hamel ME, Hardt J, Gorber SC, Tremblay M. A comparison of direct versus self-report measures for assessing physical activity in adults: a systematic review. Int J Behav Nutr Phys Act. 2008;5(1):56.

9. van Poppel MNM, Chinapaw MJM, Mokkink LB, Van Mechelen W, Terwee CB. Physical activity questionnaires for adults. Sports Med. 2010;40(7):565-600.

10. Lee PH, Macfarlane DJ, Lam TH, Stewart SM. Validity of the international physical activity questionnaire short form (IPAQ-SF): A systematic review. Int $\mathrm{J}$ Behav Nutr Phys Act. 2011;8(1):115.

11. Bull FC, Maslin TS, Armstrong T. Global physical activity questionnaire (GPAQ): nine country reliability and validity study. J Phys Act Health. 2009;6(6):790-804.

12. Baumeister SE, Ricci C, Kohler S, Fischer B, Töpfer C, Finger JD, et al. Physical activity surveillance in the European Union: reliability and validity of the European health interview surveyphysical activity questionnaire (EHIS-PAQ). Int $J$ Behav Nutr Phys Act.2016;13(1):61.

13. Finger JD, Tafforeau J, Gisle L, Oja L, Ziese T, Thelen J, et al. Development of the European health interview survey-physical activity questionnaire (EHIS-PAQ) to monitor physical activity in the European Union. Arch Public Health 2015;73(1):1-11.

14. Beaton DE, Bombardier C, Guillemin F, Ferraz MB. Guidelines for the process of cross-cultural adaptation of self-report measures. Spine. 2000;25(24):3186-91.

15. Guillemin F, Bombardier C, Beaton D. Crosscultural adaptation of health-related quality of life measures: literature review and proposed guidelines. J Clin Epidemiol. 1993;46(12):141732.

16. Faul F, Erdfelder E, Lang A-G, Buchner A. G* Power 3: A flexible statistical power analysis program for the social, behavioral, and biomedical sciences. Behav Res Methods. 2007;39(2):175-91. 
17. Faul F, Erdfelder E, Buchner A, Lang A-G. Statistical power analyses using $\mathrm{G}^{*}$ Power 3.1: Tests for correlation and regression analyses. Behav Res Methods. 2009;41(4):1149-60.

18. Craig C, Marshall A, Sjostrom M, Bauman A, Lee $P$, Macfarlane D, et al. International Physical Activity Questionnaire-Short Form. 2017.

19. World Health O. Global recommendations on physical activity for health: World Health Organization; 2010.

20. Saglam M, Arikan $H$, Savci S, Inal-Ince $D$, Bosnak-Guclu M, Karabulut E, et al. International physical activity questionnaire: reliability and validity of the Turkish version. Percept Mot Skills. 2010;111(1):278-84.

21. Chun MY. Validity and reliability of Korean version of international physical activity questionnaire short form in the elderly. Korean $\mathrm{J}$ Fam Med. 2012;33(3):144.

22. Wang C, Chen P, Zhuang J. Validity and Reliability of International Physical Activity Questionnaire-Short Form in Chinese Youth. Res Q Exerc Sport. 2013;84(sup2):S80-S6.

23. Portney LG, Watkins MP. Foundations of clinical research: applications to practice: Pearson/Prentice Hall Upper Saddle River, NJ; 2009.

24. Juniper EF. How to develop and validate a new health-related quality of life instrument. Quality of life and pharamacoeconomics in clinical trials. 1996:49-56.

25. Dewitte K, Fierens C, Stockl D, Thienpont LM. Application of the Bland-Altman plot for interpretation of method-comparison studies: a critical investigation of its practice.. Clin. Chem 2002;48(5):799-801.

26. Atkinson G, Nevill AM. Statistical methods for assessing measurement error (reliability) in variables relevant to sports medicine.. Sports Med. 1998;26(4):217-38.

27. Terwee CB, Bot SDM, de Boer MR, van der Windt DAWM, Knol DL, Dekker J, et al. Quality criteria were proposed for measurement properties of health status questionnaires.. J. Clin. Epidemiol. 2007;60(1):34-42.

28. Bofan M, Mores N, Baron M, Dabrowska M, Valente S, Schmid $M$, et al. Within-day and between-day repeatability of measurements with an electronic nose in patients with COPD. J. Breath Res.2013;7(1):017103.
29. Lowe CJM, Isaac C, Kelly $\mathrm{P}$, Barker $\mathrm{KL}$. Measuring step count: why it is important not to assume measures are reliable. Physiotherapy. 1920;110:1-4.

30. Gatti AA, Stratford PW, Brenneman EC, Maly MR. GT3X+ accelerometer placement affects the reliability of step-counts measured during running and pedal-revolution counts measured during bicycling.. J. Sports Sci. 2016;34(12):1168-75.

31. Scuderi GR, Sikorskii A, Bourne RB, Lonner JH, Benjamin JB, Noble PC. The Knee Society short form reduces respondent burden in the assessment of patient-reported outcomes. Clin Orthop Relat Res. 2016;474(1):134-42.

32. Turner D, Schünemann HJ, Griffith LE, Beaton DE, Griffiths AM, Critch JN, et al. The minimal detectable change cannot reliably replace the minimal important difference. J. Clin. Epidemiol.2010;63(1):28-36. 